\title{
EEG-Wave Metamorphosis Available for Clinical Electroencephalography : A Preliminary Report
}

\author{
By \\ Toyoji Wada, Akira Goto, Yutaka Fukushima and \\ Takamaro Shimizu* and Takeshi Ohya** \\ From* the Department of Neuropsychiatry, Hirosaki University
school of Medicine, Hirosaki. Director: Prof. T. Wada
and** Nihon Koden EEG Inst. Co.. President: Dr. Y. Ogino
}

(Received for publication, October 21, 1961)

By means of the so-called automatic EEG-analyser ${ }^{1 / 2) 3}$ ) having been introduced in both clinical and experimental fields at the present day, we are able to see in formations of EEG components from various points of view, either quantitatively or qualitatively. As far as the clinical electroencephalography is concerned, however, it seems impossible to deny there are several short points when those analysers are applied in clinical usage.

Although instantaneously analysed values due to the common automatic EEG analyser are usually informed us through an instantaneous and quantitatative expression of each limited frequency band, the correlation between the original EEG pattern and those data, especially the transformation of those values into simple quantitative data being convenient or immediately available for clinical application seems rather difficult at least at the present stage of knowledge. In clinical EEG, not only instantaneous values in a certain limited time of duration of recording (viz., an averaged value), but sporadic or transient outbursts of single discharge, are important factors for evaluation of EEG recorded; in other words, the characteristics of each wave component, including wave figure of single activity, as well as averaged values of amplitude and frequency, present data necessary for EEG evaluation.

Indeed, especially in clinical fields, almost all electroencephalographers dealing with so many EEGs every day, have no sufficient time of so long duration for evaluation of each EEG recorded from patients through application of theoretically designed parameters. In practical point of view, they usually seek after abnormal signs occurring as sporadic or paroxysmal changes. Therefore, it might be rather desirable if we could find only abnormal EEG signs out of the normal pattern at a glance, even if those signs occur continuously, sporadically or

和田豊治, 後藤 昭, 福島 裕, 清水隆䗪, * **大谷 武 
paroxysmally. In fact, can we who have been engaged in clinical examination every day, deny to be tended to seek after not normal alpha activity but other abnormal discharge itself? Toward such a purpose, some contributions have been made by the authors, and certain results are described in this paper.

\section{RESULTS}

At first we have designed such a filter as shown in Fig. 1 in order to eliminate, at first, the normal alpha wave from EEG pattern, and then other filters for the delta and beta activities. Applied those filters to out-put of EEG apparatus, theoretically the EEG figure should be metamorphosed and also recorded as shown in Fig. 2. If it were recorded through the alpha band filter, then there might be obtained the record without any alpha rhythm and only other abnormal components belonging to the beta or delta frequency. A sample of experimental result is represented in Fig. 3 which had been recorded through an oscillator applied as a generator with frequency within limits of alpha rhythm.

Next, several data obtained practically from patients are indicated. Fig. 4 is a record obtained from a normal subject, in which the most significant change is recognizable in the third curve, viz. the alpha-excluded line. Fig. 5 is a record during a subclinical seizuer pattern of $3 / \mathrm{sec}$. rhythmic spike-and-wave complex, in which no significant variation is recognizable in the alpha-excluded line, but spike-component is stressed in the delta-excluded and slow wave component in the beta-exlcuded. Fig. 6 shows a record from a patient with focal brain damage ; in the original EEG there are slow irregular activity and sporadic small spike, but in the metamorphosed record, a significant slowing observed is in the

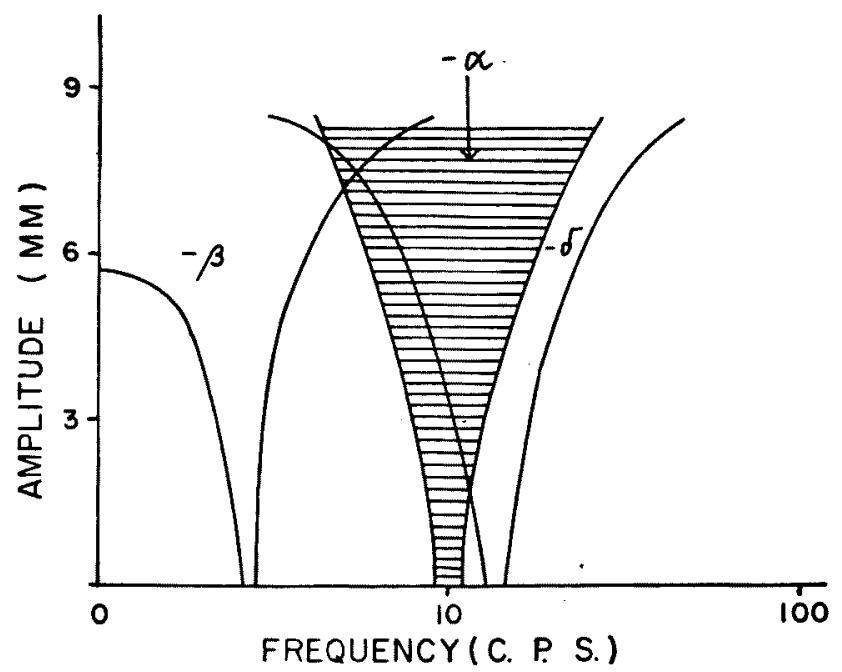

Fig. 1. Character of designed filters. 


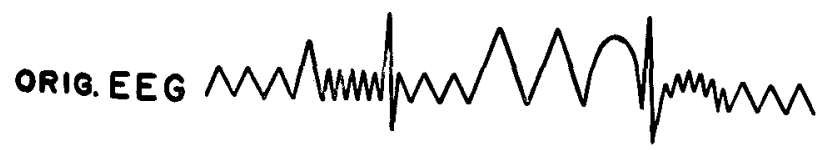
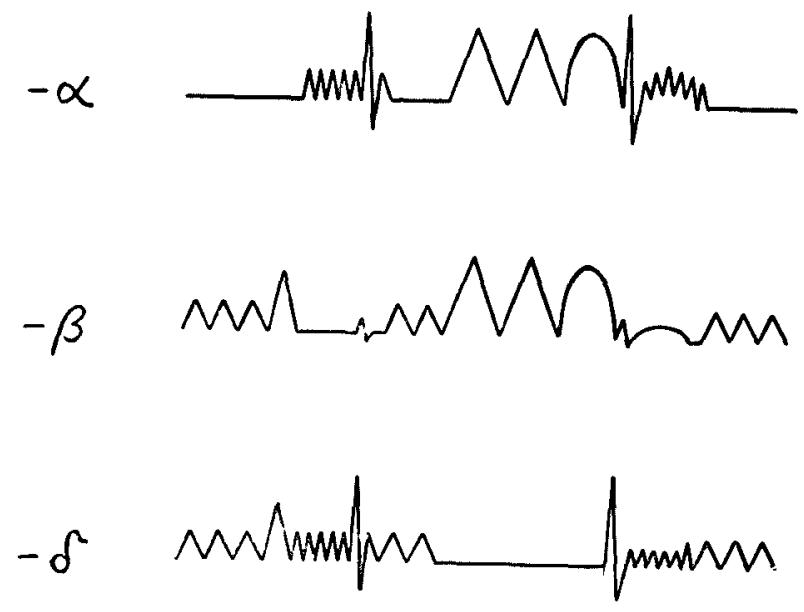

Fig. 2. Principal schema of theoretical record with exclusion of each frequency band.
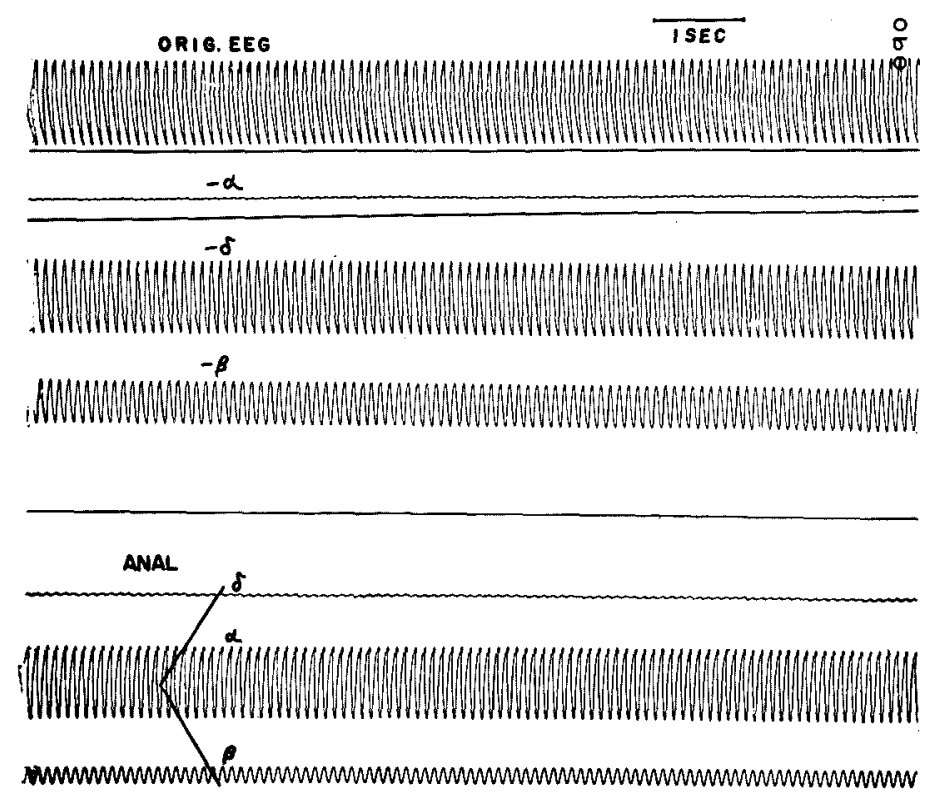

Fig. 3. Result in case of artificially applied oscillator with frequency of $10.5 \mathrm{c} / \mathrm{s}$. The lower three curves show the result of simultaneously analysed data or instantaneous value in delta-, alpha- and beta-band. 


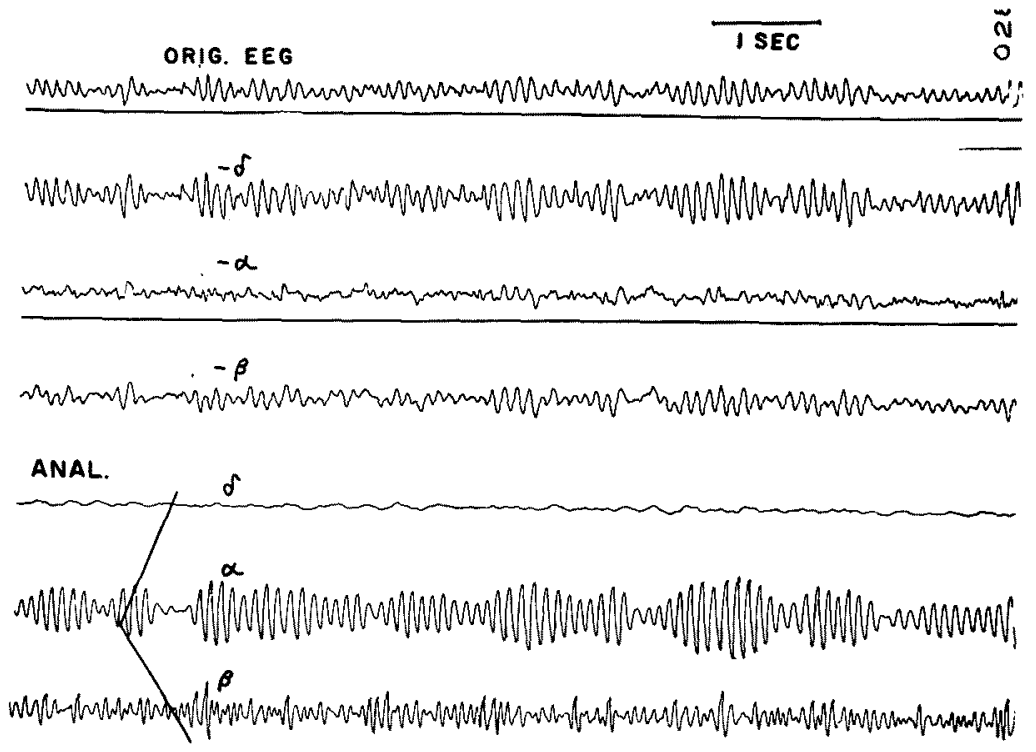

Fig. 4. A record obtained from a normal subject with rich alpha activity.

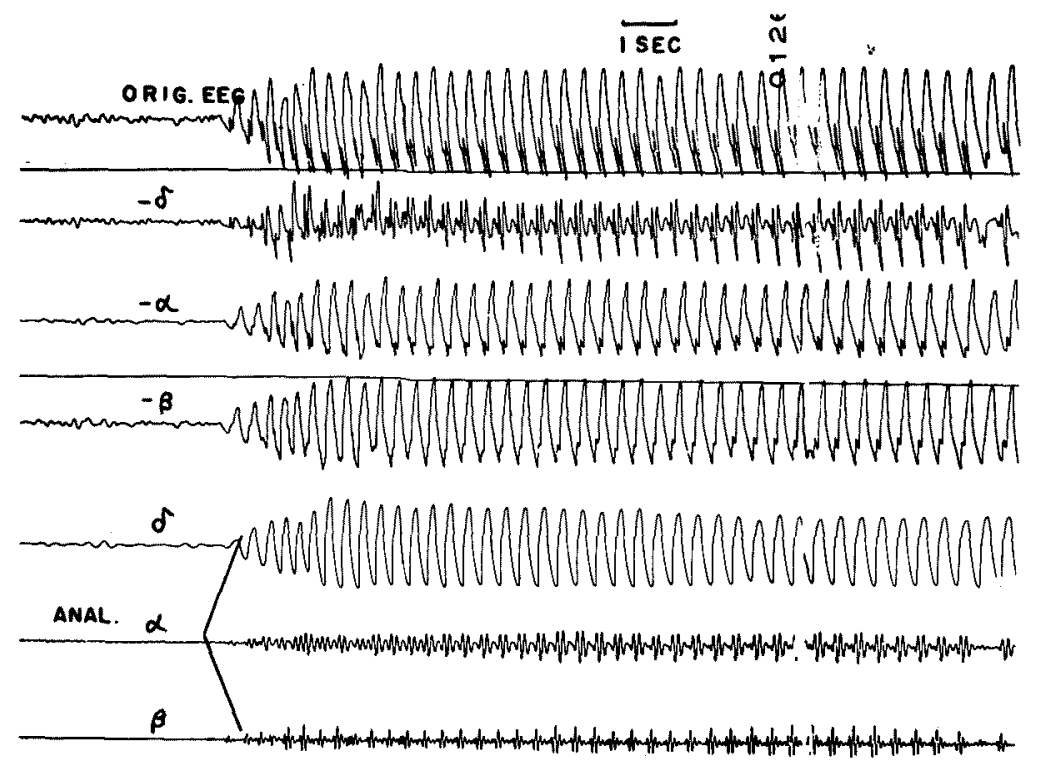

Fig. 5. A record during subclinical seizure pattern of petit mal absence. 


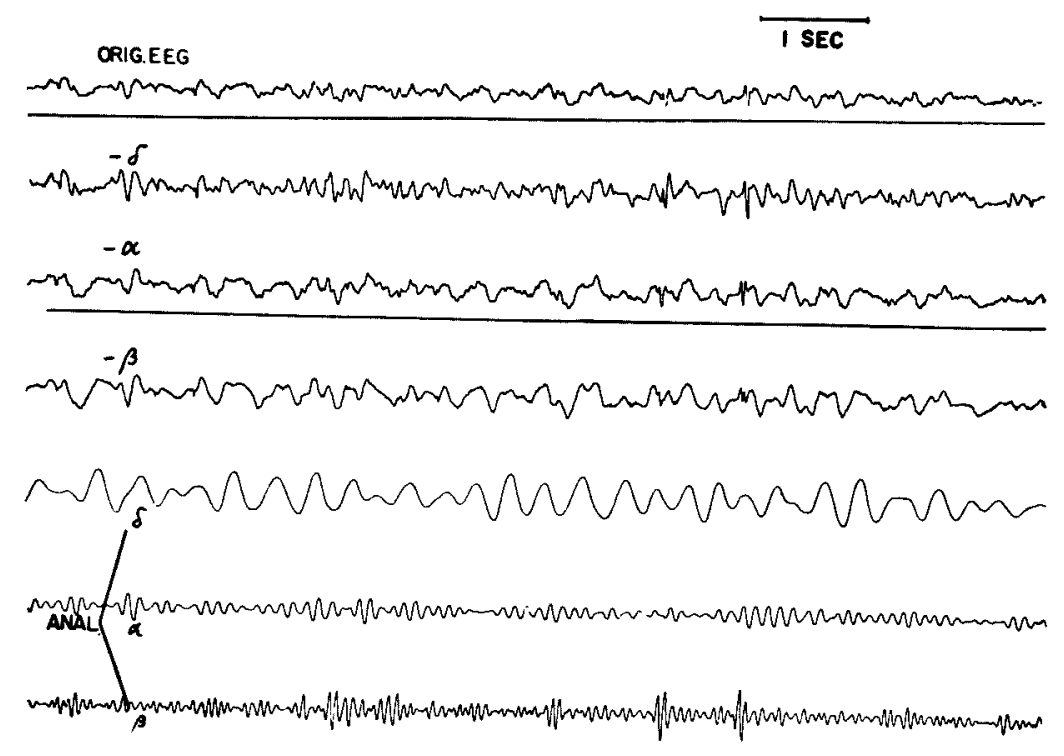

Fig. 6. A record obtained from a patient with focal EEG signs.

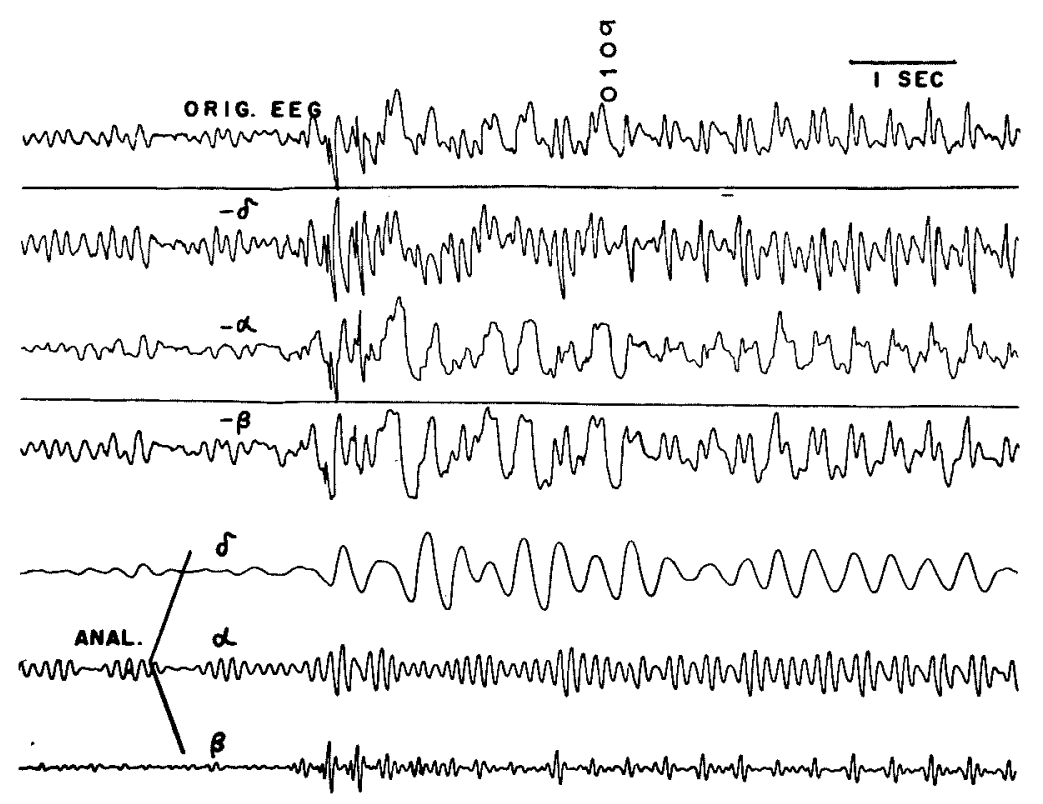

Fig. 7. A record during epileptic discharges with spike- and sharp-wave complex of semirhythmic nature. 
beta-excluded pattern and, conversely, a remarkable spike factor in the deltaexcluded pattern. Fig 7. indicates a record from a epileptic patient with paroxysmal bursts of sharp-and-wave complex of semirhythmic nature, in which both slow wave and fast activity are clearly recognized in the alpha-excluded record.

\section{COMMENT AND CONCLUSION}

As described above, we have made efforts to obtain EEG-figure which lacks the normal alpha activity and shows only other abnormal factors with stressed picture. This metamorphosis of EEG-figure is easy in theoretical sense, however its practice, as shown in a series of sample, seems difficult, if we seek after its complete establishment. This is, of course, due to the difficulty in designation of any suitable and complete filter, because EEG activity changes its component without interruption, not only figures but even in both amplitude and frequency. However, the principal idea to metamorphose the EEG-wave might be necessary in clinical electroencephalography, because it seems much more convenient for finding abnormal discharges, even due to the author's insufficient results. Accordingly, it is obviously necessary to have further progress in design of the filter-circuit itself. Though in this preliminary report, only a principal idea and some experimental results have been mentioned, but this is due to the desire for a further progress in clinical electroencephalography.

\section{References}

1) Grass, A.M. \& Gibbs, A.F., J. Neurophysiol., 1938, 1, 521.

2) Sakamoto, T., Proceeding the IXth Annual Meeting of the Japan EEG Society, 1960, 27.

3) Wada, T. et al., Proceeding the IXth Annual Meeting of the Japan EEG Society, $1960,27$. 